\title{
Optimization and Numerical Analysis of the Ricochet of Conical Nose Projectile in the Collision with Ceramic- Aluminum Armor
}

\author{
Bahman Salimi $^{1^{*}}$, Khodadad Vahedi ${ }^{1}$, Amin Moslemi Petrudi ${ }^{2}$, Masoud \\ Rahmani $^{2}$
}

${ }^{1}$ Department of Mechanical Engineering,

IHU University, Tehran, IRAN

${ }^{2}$ Department of Mechanical Engineering,

Tehran University, Tehran, IRAN

*Corresponding Author

DOI: https://doi.org/10.30880/jamea.2020.01.02.006

Received 30 June 2020; Accepted 18 August 2020; Available online 31 October 2020

\begin{abstract}
The anti-armored bullets have more kinetic energy due to additional mass than ordinary bullets which increases their likelihood function of penetration for a different target. It is necessary to seek solutions to reduce the possibility of penetration of this type of bullets. One of the most important parameters influencing penetration is projectile impact velocity. The mechanism of penetration varies in different velocity ranges. In this paper, the phenomenon of buckling steel cone with a nose cone in a collision with ceramic targets was investigated by the explicit finite element method using LS-Dayna software. The numerical simulation shows acceptable accuracy after comparing the results with previous research. In this study, the critical angle of ricochet at a different velocity ranging from $700 \mathrm{~m} / \mathrm{s}$ to $1000 \mathrm{~m} / \mathrm{s}$ and optimization of the optimal thickness ratio of ceramic/metal targets has been considered. According to the simulation outputs and analytical relations, it is clear that the critical ricocheting angle has increased with increasing velocity and the probability of projectile penetration is higher. The results also show that in the case of an oblique collision at a certain angle, the projectile velocity decreases with increasing target thickness with no drastic changes in the directional angle. As the angle of oblique increases, the amount of penetration in the target decreases. For long rod projectiles, the reduction in kinetic energy at the same collision velocities is not much different for both vertical and oblique collision modes. The erosion of the projectile mass in oblique collisions is less than in vertical collisions at the same time.
\end{abstract}

Kevwords: Ricochet. Numerical simulation. Anti-armored bullets. Alumina ceramic. LS-Davna software.

\section{Introduction}

Penetration mechanics is a subset of impact mechanics which studies the response of materials and structures to very intense dynamic loads in a short time. The study of penetration mechanics was studied by researchers from various military and civilian agencies. Potential military applications include the design and construction of impact-resistant structures including different types of armor, jackets, bulletproof panels, trenches, rocket, and ballistic missiles design. Furthermore, several examples of penetration mechanics application include the protection of satellites and spacecraft in the event of meteorite collisions, the protection of nuclear reactors and vehicles for the transport of hazardous materials, the welding and explosive shaping of parts, and the reinforcement of car and aircraft fuselages against various objects.

One of the critical aspects to consider in the design of armored vehicles is the ricochet projectile. The task to predict the ricochet behavior of a projectile is challenging owing to numerous parameters involved. Numerous studies have been 
conducted in this area, considering the importance of this issue in the military industry. Jonas and Zukas [1] examined the mechanism of the ricochet phenomenon in several numerical simulations and tests. The study concludes that with increasing collision velocities, tensions increased, and in a particular case, an excessive increase in these tensions led to the displacement of the target's hydrodynamics and projectiles near their frontier. Tate [2] presented an analytical model for the ricochet problem. Gupta et al. [3] studied the effect of the shape of the head of long rod projectiles on regular collisions with the thin metal walls. They observed that the ballistic performance of the cone projectiles is better than flat head projectiles. The phenomenon of riding on ceramic surfaces was examined numerically by Wang et al. [4]. They concluded that at small angles, the projectile hits the direction after the infiltration in the opposite direction and tends to be vertical towards the target. However, with increasing angle of impact, from certain angles to the next, the projectile turns during the impact and in the direction of the agreement, and as a result, the angle of inclination increases, which is a useful phenomenon to increase the efficiency of armored ceramics. Marina et al. [5] explored numerically and experimentally the ricochet projectile in dealing with steel plates. In ceramic, because of the brittle structure and the type of impact damage, the penetration resistance is not constant and can vary depending on the conditions and velocity of impact and penetration and even the backing layer conditions. Lee et al. [6] reviewed the ricochet of a tungsten heavy alloy long-rod projectile from deformable steel plates. Moslemi Petrudi et al. [7] study the effect of a blunt projectile having a specified speed penetrating a ceramic-aluminum target at angles of $0^{\circ}, 15^{\circ}, 30^{\circ}$, and $45^{\circ}$. It can be seen that the critical ricochet angle in ceramic targets with an aluminum backing plate has not been considered in previous studies, and this effect will be investigated in detail in this study through numerical simulation.

Liu et al. [8] investigate the mechanical characteristics, including tensile and flexural properties of wood, ceramic, copper, aluminum, and carbon fiber-based polylactic acid (PLA) composites. The effects of different PLA composites, build orientations, and raster angles on mechanical responses are compared and analyzed in detail. Islam et al. [9] proposed three constitutive relations generally employed to study deformations of a ceramic are due to Johnson and Holmquist, generally abbreviated as JH1, JH2, and JHB. The deformation problem was implemented in the computational algorithm using Smoothed Particle Hydrodynamics (SPH) basis functions and a pseudo-spring technique to simulate the initiation and propagation of material failure. Rodríguez et al. [10] investigate two different composed armors that have been analyzed for comparison: one consisting of a ceramic layer and an aluminum 2024-T3 alloy plate, and the other combining ceramic and aramid plates. As the main conclusion, it has been confirmed the higher performance of aramid fibers over aluminum in aircraft ballistic protection, both in terms of protection and weight reduction. Alumina ceramic is an excellent choice for armor construction due to its relatively good ballistic properties and lower cost. In this paper, a numerical study of the phenomenon of rustling a special type of anti-armored steel projectiles in dealing with alumina ceramic armor with an aluminum support plate of 2024 has been investigated. The point to be considered is that in ceramics, due to the brittle structure and the specific type of damage due to impact, the penetration resistance is not constant and can change depending on the conditions and speed of impact and penetration and even the conditions of the backing layer. In the following, the issue of the optimal thickness of the ceramic and aluminum sections is discussed.

\section{Problem Theory}

A ricochet occurs if the bullet projectile does not penetrate or stay inside the target after a collision [12]. For a certain velocity of the projectile, there is a minimum angle in which the projectile ricochets. Tate [2] stated that due to the high velocity of the bullet, shape and the displacement of the projectile material from the main axis of the projectile, it is possible to assume the projectile's penetration directly.

Consider a schematic of a projectile striking an oblique plate shown in Fig. 1. The projectile's front surface will be eroded in a complicated way during penetration with the target. An approximation of the physical problem can be obtained by making the following assumptions:

1. The deacceleration of the projectile during infiltration is considered.

2. The erosion of the projectile's front surface is equal to the erosion rate in the stable hydrodynamic phase of penetration.

3. The effective tension in the end part is considered to be the same as the hydrodynamic resistance of the projectile material.

When a projectile strikes the target with sufficiently high velocity, both projectile and target will deform in a hydrodynamic behavior. The modified hydrodynamic approximation of the problem is given in the form of the Bernoulli equation as follow [13][14]:

$$
\frac{1}{2} \rho_{p}(V-U)^{2}+Y_{P}=\frac{1}{2} \rho_{t} U^{2}+R_{t}
$$

In this equation, $U$ is the velocity of projectile penetration, $Y_{P}$ and $R_{t}$ is the projectile and target's resistance to penetration, $\rho_{p}$ and $\rho_{t}$ are the projectiles and target density, $L$ and $D$ are the projectile's length and diameter, and $V$ is the initial velocity of the projectile. As a result, the penetration velocity, $U$ is obtained by rearranging Equation (1) into: 


$$
U=\frac{\rho_{p} V-\sqrt{\rho_{p}^{2} V^{2}-\left(\rho_{p}-\rho_{t}\right)\left(\rho_{P} V^{2}+2\left(Y_{P}-R_{t}\right)\right)}}{\rho_{p}-\rho_{t}}
$$

For deep penetration, the resistance strength of projectile should be greater than projectile's material strength at $R_{t}=$ $3 Y_{P}$. A strong target will not suffer any significant deformation unless the initial velocity $V$ exceeds a critical value $V_{c r i t}$. Thus, the ricochet can occur if the target plate is oblique to the projectile trajectory, and the initial velocity did not exceed the critical velocity. The estimation of the critical velocity can be obtained from Equation (1) by setting penetration velocity, $U$ to zero which yields:

$$
V_{c r i t}=\left[\frac{2\left(R_{t}-Y_{P}\right)}{\rho_{p}}\right]^{\frac{1}{2}}
$$

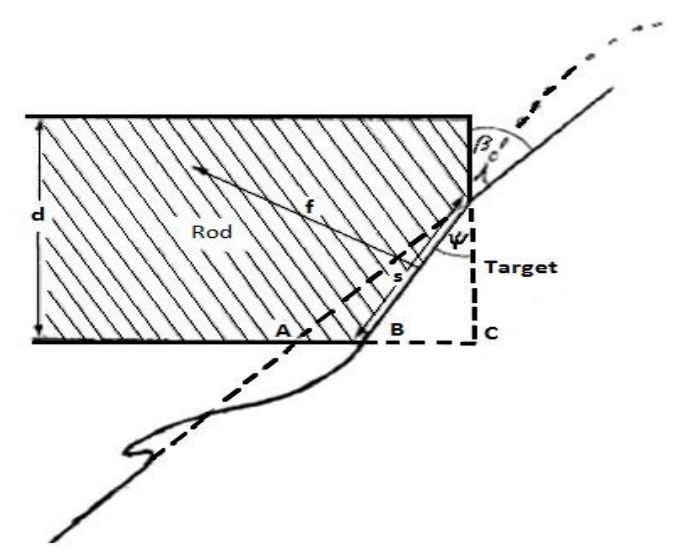

Fig. 1 - Schematic of the projectile and target after collision [2]. AB $=\mathrm{Ut}$ and $\mathrm{AC}=\mathrm{Vt}$.

At velocities above the critical velocity, the projectile starts to penetrate the target but at the same time, it rotates.

Let the length of the projectile to be $l$ and the side of the square cross-section in Fig. 1 to be $d$. Consider the measurement of time is taken from the instant the projectile striking the target and let the obliquity of impact be $\beta$. A few moments after the initial impact, the length of the eroded surface $S$, can be approximated as:

$$
S=\frac{(V-U) t}{\sin \psi}
$$

As a result, the force $f$ normal to the eroding surface is equal to:

$$
f=Y_{P} d S
$$

and the moment created by this force around the center of gravity of the projectile is given as follows:

$$
C=\frac{1}{2} f l \sin \psi
$$

Assuming that the projectile does not deflect too much off course, the minimum estimate of the time $t_{m}$ before the whole projectile engages the target yields:

$$
t_{m}=D \tan \frac{\beta}{V}
$$

At this time, moment $C$ has induced a rotary motion to the projectile such that the yaw angle $\theta$ satisfies the following equation:

$$
\ddot{\theta}=\frac{12 C}{M\left(l^{2}+d^{2}\right)}
$$

In the above relation, $M$ is the projectile mass, and $\theta$ is the angular displacement of the projectile. Ricochet occurs when $\dot{\theta}>2 V \cot \frac{\beta}{l}$. Tate [2] proposed further expansion of Equation (8) which yields the following results: 


$$
\ddot{\theta}=A t
$$

where,

$$
A=6 Y_{P} \frac{(V-U)}{\rho_{p} d}\left(l^{2}+d^{2}\right)
$$

By integration Equation (9) yields:

$$
\dot{\theta}=\frac{1}{2} A t^{2}
$$

Finally, the ricocheting angle is defined as follows:

$$
(\tan \beta)^{3}>\frac{2}{3} \frac{\rho_{p} V^{2}}{Y_{P}}\left(\frac{l^{2}+d^{2}}{l d}\right)\left(\frac{V}{V-U}\right)
$$

The expression (11) at the high collision velocities tends to follow the following form:

$$
(\tan \beta)^{3}>\frac{2}{3} \frac{\rho_{p} V^{2}}{Y_{P}}\left(\frac{l^{2}+d^{2}}{l d}\right)\left[1+\left(\frac{\rho_{p}}{\rho_{t}}\right)^{\frac{1}{2}}\right]
$$

In 1989, Rosenberg [11] presented a model for analyzing a critical ricochet angle, which, unlike Tate [2] theory, was no longer considered rigid. Here, the ricochet is defined according to Fig. 1:

$$
U=\frac{b}{t}=\frac{x \tan \beta}{t}
$$

Contrary to the analytical model of Tate [2], it is assumed that the amount of force depends on the applied pressure from the target material to the projectile. The force can be calculated as follows:

$$
f=R_{t} S D
$$

The vertical impact force on the projectile is:

$$
\begin{gathered}
I=\int_{0}^{t_{m}} f \sin \psi d t=\frac{R_{t} D^{3}(V-U)}{2 V^{2}} \tan \beta \\
V_{T}=\frac{R_{t}}{\rho_{p} V^{2}}\left(\frac{V-U}{V+U}\right) V \cdot \tan \beta
\end{gathered}
$$

The projectile ricochet angle satisfies the following condition:

$$
\tan \beta>\frac{V}{V_{T}}
$$

Therefore, considering this condition in the above equation, the critical angle of ricocheting in the Rosenberg [14] model is obtained from the following relationship:

$$
(\tan \beta)^{2}>\frac{\rho_{p} V^{2}}{R_{t}}\left(\frac{V+U}{V-U}\right)
$$

The conditions of this model are different in several cases with the Tate model [2]. In the Rosenberg model [14], the critical angle of ricochet depends on the target's resistance, whereas in the Tate model [2], this angle depends on the resistance of the projectile material. This happens due to the consideration of projectile erosion in the Rosenberg model [14] while the penetration model in Tate [2] is considered the projectile material to be rigid. As a result, the parameters of projectile length and diameter do not appear in the critical angle of ricocheting relationship in the Rosenberg theory [11].

\section{Numerical Simulation}

In this part of the paper, Solidworks design software has been used to model the projectile geometry and target. In this application in three a separate part is designed for the projectile, the ceramic plate, and the backing layer. Then these three parts are assembled and the dimensions of ceramic and aluminum are $50 \times 50 \mathrm{~mm}$, and their thickness is 10 and 6 
mm. The Hyper Mesh software, which is software powerful in the field of high-quality mesh, has been used. The quad type and the number of elements according to Table 1 are specified below. The critical point in choosing the element is that after meshing, the grid should be as uniform as possible. The smaller the number of meshes, the higher the accuracy of the software in simulating ricocheting at different velocities ranging from $700 \mathrm{~m} / \mathrm{s}$ to $1000 \mathrm{~m} / \mathrm{s}$. These velocities are average and are due to the propagation behavior of elastoplastic waves in this velocity range. For this purpose, a steel projectile with a diameter of $7.62 \mathrm{~mm}$ and a length of $25.5 \mathrm{~mm}$ made of ceramic material is used with a backing of aluminum metal. The thickness of the ceramic is $10 \mathrm{~mm}$ and the thickness of aluminum is $6 \mathrm{~mm}$. A schematic of the meshed sample is shown in Fig. 2. The type and characteristics of the support and the projectile and target models for simulating are presented in Table 1. The specifications for materials used in the simulation are presented in Table 2-4.

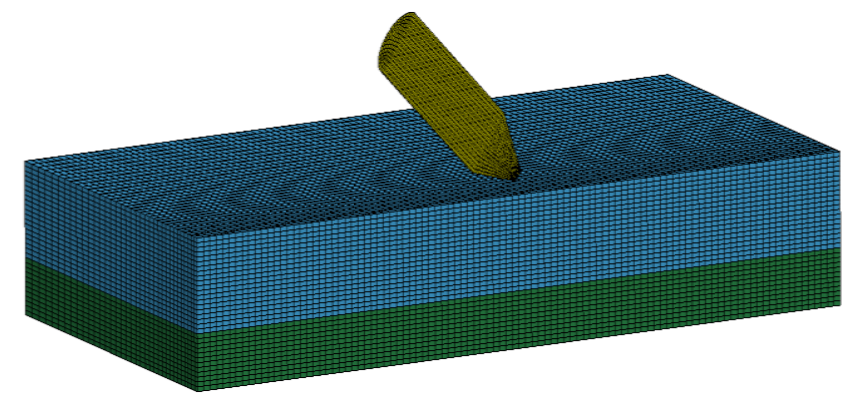

Fig. 2 - Elemental image of the projectile and the target of simulating the phenomenon of ricochet

Table 1 - Boundary conditions and simulation models [15]

\begin{tabular}{cccccc}
\hline Material & Element type & Material model & Equation of state & $\begin{array}{c}\text { Number of } \\
\text { elements }\end{array}$ & $\begin{array}{c}\text { Boundary } \\
\text { conditions }\end{array}$ \\
\hline Ceramic & Cubic & Johnson Holmquist & - & 140000 & Clamp \\
Steel & Cubic & Plastic_Kinematic & Mie-Gruneisen & 8950 & Free \\
Aluminum & Cubic & Johnson Cook & Mie-Gruneisen & 60000 & Clamp \\
\hline
\end{tabular}

Table 2 - Proportional steel material model [15]

\begin{tabular}{lcc}
\hline Parameter & Amount & Symbol (unit) \\
\hline Density & 7800 & Ro $\left(\frac{\mathrm{kg}}{\mathrm{m}^{3}}\right)$ \\
Shear modulus & 80 & $\mathrm{G}(\mathrm{GPa})$ \\
Modulus of elasticity & 210 & $\mathrm{E}(\mathrm{GPa})$ \\
Poisson's ratio & 0.31 & $\mathrm{PR}$ \\
Initial yield strength & 507 & $\mathrm{~A}(\mathrm{MPa})$ \\
Hardness constant & 320 & $\mathrm{~B}(\mathrm{MPa})$ \\
Strain rate constant & 0.28 & $\mathrm{C}$ \\
First failure parameter & 0.15 & $D_{1}$ \\
Second failure parameter & 0.72 & $D_{2}$ \\
Third failure parameter & 1.66 & $D_{3}$ \\
Fourth failure parameter & 0.005 & $D_{4}$ \\
Fifth failure parameter & -0.84 & $D_{5}$ \\
Gruneisen parameter & 1.70 & $\Gamma$ \\
\hline
\end{tabular}


Table 3 - Specifications of the 6061 aluminum material used in simulation [15]

\begin{tabular}{lcc}
\hline Parameter & Amount & Symbol (unit) \\
\hline Density & 2700 & $\mathrm{Ro}\left(\frac{\mathrm{kg}}{\mathrm{m}^{3}}\right)$ \\
Shear modulus & 26 & $\mathrm{G}(\mathrm{GPa})$ \\
Modulus of elasticity & 68 & $\mathrm{E}(\mathrm{GPa})$ \\
Poisson's ratio & 0.33 & $\mathrm{PR}$ \\
Initial yield strength & 324 & $\mathrm{~A}(\mathrm{MPa})$ \\
Hardness constant & 114 & $\mathrm{~B}(\mathrm{MPa})$ \\
Strain rate constant & 0.002 & $\mathrm{C}$ \\
First failure parameter & -0.77 & $D_{1}$ \\
Second failure parameter & 1.45 & $D_{2}$ \\
Third failure parameter & -0.47 & $D_{3}$ \\
Fourth failure parameter & 0 & $D_{4}$ \\
Fifth failure parameter & 1.6 & $D_{5}$ \\
Gruneisen parameter & 2.14 & $\Gamma$ \\
\hline
\end{tabular}

Table 4 - Specifications of the $\mathrm{Al}_{2} \mathrm{O}_{3}$ ceramic material model used in simulation [15]

\begin{tabular}{lcc}
\hline Parameter & Amount & Symbol (unit) \\
\hline Density & 3800 & Ro $\left(\frac{\mathrm{kg}}{\mathrm{m}^{3}}\right)$ \\
Shear modulus & 0.989 & $\mathrm{G}(\mathrm{GPa})$ \\
Normal initial resistance & 0.989 & $\mathrm{~A}(\mathrm{GPa})$ \\
Normal failure resistance & 0.77 & $\mathrm{~B}(\mathrm{GPa})$ \\
Constant strain rate & 0 & $\mathrm{C}$ \\
Reference strain rate & 1 & $\mathrm{EPSI}\left(\mathrm{s}^{-1}\right)$ \\
Maximum tensile strength & 0.15 & $\mathrm{~T}(\mathrm{GPa})$ \\
Maximum normal failure resistance & 0.5 & $\mathrm{SFMAX}$ \\
Hugoniot elastic limit & 5.9 & $\mathrm{HEL}(\mathrm{GPa})$ \\
Pressure component in Hugoniot elastic limit & 2.2 & $\mathrm{PHEL}(\mathrm{GPa})$ \\
Bulk modulus & 200 & $K_{1}(\mathrm{GPa})$ \\
Second pressure factor & 0 & $K_{2}(\mathrm{GPa})$ \\
Third pressure factor & 0 & $K_{3}(\mathrm{GPa})$ \\
Failure criterion & 1.5 & FS \\
\hline
\end{tabular}




\section{Results and Discussion}

One of the main parts of any research work is the validation of the results with other researchers' research. In this section, we use numerical solution validation for comparing the amount of backup layer displacement with experimental values. Fig. 3 shows a comparison between the results of the simulation and the experimental results given in [16]. The values used to compare the displacement of the aluminum layer with the source [16] are also reported in Table 5. It can be seen that the error rate is acceptable. In the following, it is necessary to consider the critical angle in which the projectile penetrates. In Fig. 4, the projectile with $1000 \mathrm{~m} / \mathrm{s}$ velocity penetrates the surface at an angle of $30^{\circ}$ from the normal line to the surface of the penetration. It is observed in Fig. 5 that for an angle of $67^{\circ}$, the projectile ricochets after collisions. In both simulations, the thickness of the ceramic is $10 \mathrm{~mm}$ and the aluminum thickness of the back layer is $6 \mathrm{~mm}$.

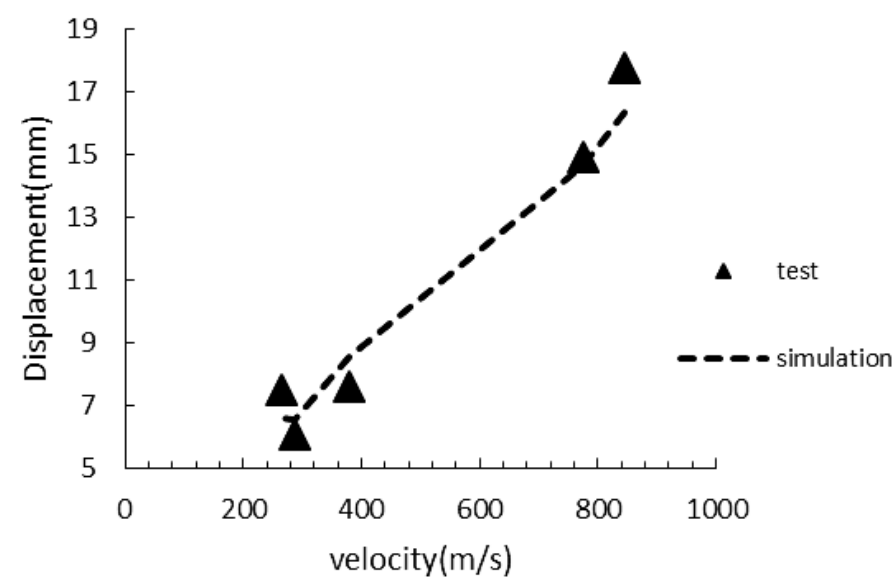

Fig. 3 - Comparison between simulation results and laboratory results [16].
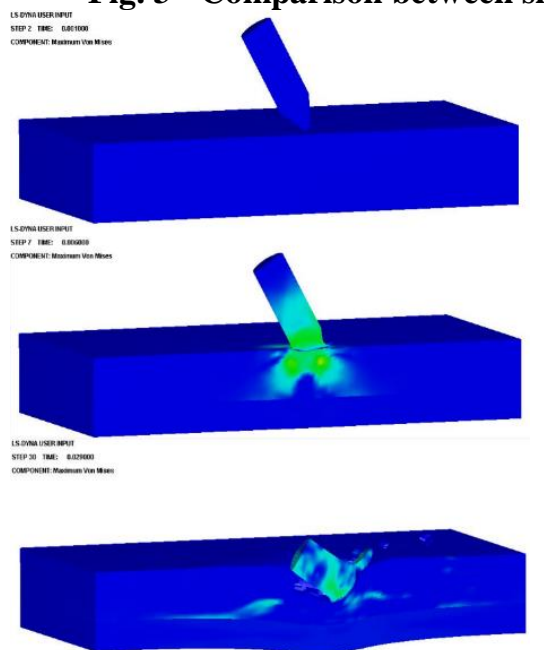

Fig. 4 - The projectile penetration at $1000 \mathrm{~m} / \mathrm{s}$ and $30^{\circ}$ to the normal line on the surface.
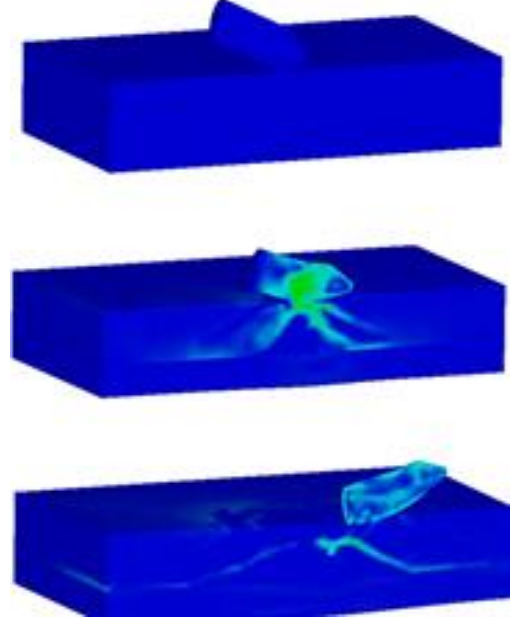

Fig. 5 - The projectile ricochets at the velocity of $1000 \mathrm{~m} / \mathrm{s}$ with $67^{\circ}$ inclination from the normal line on the surface.

Table 5 - Comparison of numerical and test values [16]

\begin{tabular}{ccccc}
\hline $\begin{array}{c}\text { Test } \\
\text { number }\end{array}$ & $\begin{array}{c}\text { Collision velocity } \\
(\mathbf{m} / \mathbf{s})\end{array}$ & $\begin{array}{c}\text { Numerical results } \\
(\mathbf{m m})\end{array}$ & $\begin{array}{c}\text { Experimental results } \\
(\mathbf{m m})\end{array}$ & $\begin{array}{c}\text { Percentage error } \\
(\boldsymbol{\%})\end{array}$ \\
\hline 1 & 775 & 14.67 & 14.91 & 1.61 \\
2 & 844 & 16.35 & 17.77 & 7.99 \\
3 & 378 & 8.58 & 7.62 & 12.60 \\
4 & 266 & 6.41 & 7.33 & 12.55 \\
5 & 287 & 6.57 & 6.11 & 7.53 \\
\hline
\end{tabular}


In Fig. 6. and Fig. 7. variations in the projectile velocity at collision angles of $30^{\circ}$ and $67^{\circ}$ are given. It can be seen that the critical angle can also be calculated using the analytical relation extracted in Section 2 . It should be noted that when the projectile hits the surface, the projectile is eroded, and the projectile mass also changes with time, and the projectile erosion also depends on the angle of impact, the projectile's velocity, and other parameters. In Fig. 8. and Fig.9, the kinetic energy changes observed by simulation are visible.

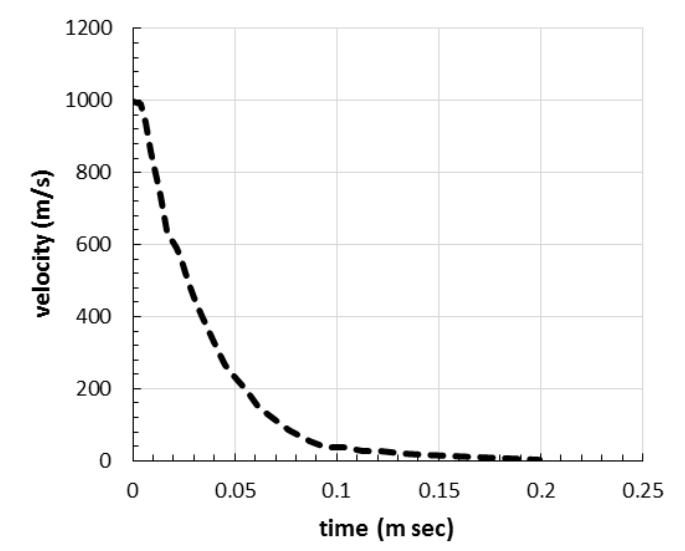

Fig. 6 - The variation of the projectile's velocity at penetration angles of $30^{\circ}$ compared to the normal

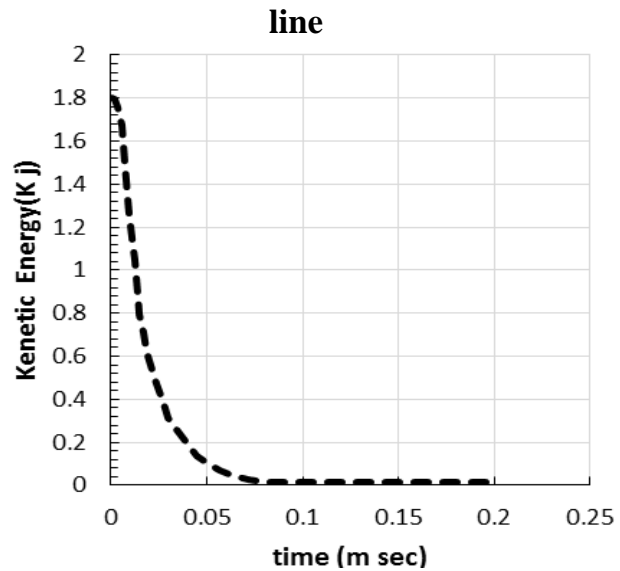

Fig. 8 - The kinetic energy changes of the projectile $30^{\circ}$

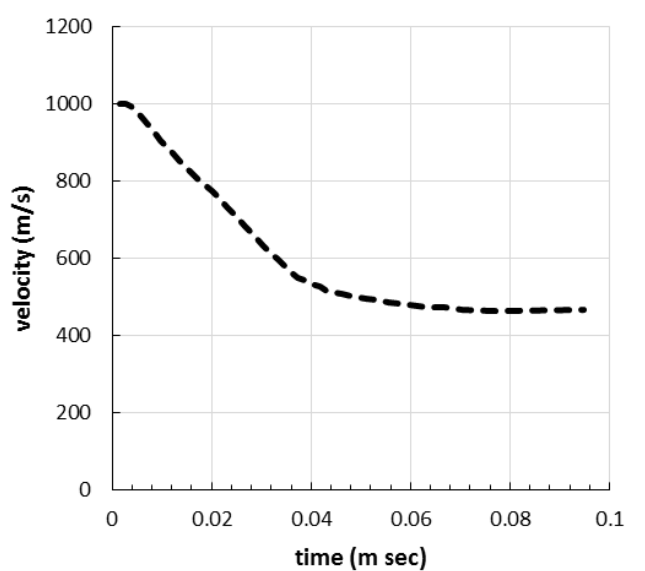

Fig. 7 - The variations in the projectile's velocity at a steady angle of $67^{\circ}$ from the normal line

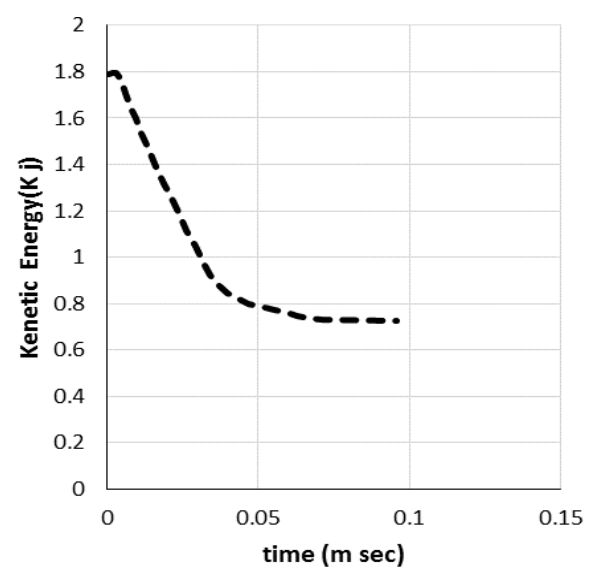

Fig. 9 - The kinetic energy changes of the projectile with a collision angle of $67^{\circ}$

Figures 6 and 8 show that the bullet hit the target at a 30-degree angle and got stuck in the target, with zero kinetic energy. However, in Figures 7 and 9, the bullet hit the target at a 67-degree angle to the vertical line (A line perpendicular to the target page), and after ricocheting, it continued to move at a speed of 430 meters per second. According to the numerical studies presented to estimate the ricochet angle, it is concluded that at higher impact velocities, the values of the critical angle for ricochet increase with a higher slope. Figure 10 shows a comparison between the results of the modified analytical relation for ceramics (Equation (18)) and numerical simulation for the critical angle of ricochet. This diagram is obtained using several simulations and can be used to obtain the approximate angle of ricocheting at different impact velocities for the aluminum ceramic particle with the previously mentioned specifications. According to Figure 10 and the comparison of simulation results with analytical results, the results are close and acceptable. 


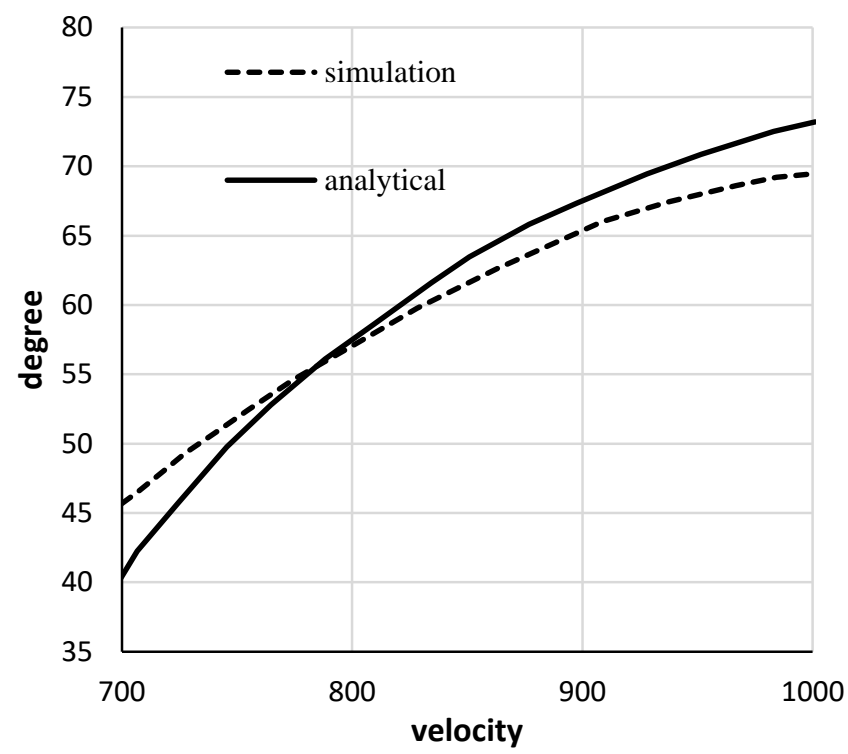

Fig. 10 - Comparison of the critical angle of ricocheting obtained from the analytical Equation (18) and numerical simulation

\subsection{Optimization of optimal thickness ratio of ceramic/metal targets}

In this section, we will examine the optimal thickness ratio of ceramic numerically to supporting metal to achieve the highest performance of the armor. In this study, the projectile is thrown at three different velocities of $700 \mathrm{~m} / \mathrm{s}, 1000$ $\mathrm{m} / \mathrm{s}$, and $1400 \mathrm{~m} / \mathrm{s}$ towards a ceramic/metal target with a total thickness of $10 \mathrm{~mm}$. Fig. 11-13 show the optimum ceramic thickness (in millimeters) at various velocities and angles. The mark in each figure represents the optimum ceramic thickness at that angle and the collision velocity which is calculated from Equation (1). The angles are measured relative to the line perpendicular to the surface. According to Fig. 11. to Fig. 13. it is clear that with increasing collision rate, the role of the ceramic has been enhanced to improve the armor performance and The optimal size of the ceramic has been increased, on the other hand, with the increase in the angle of the collision, the increasing role of the supporting aluminum sheet and the thickness of the ceramic plate decreased. When the projectile collision the surface, the projectile is eroded and the mass of the projectile changes with time, which also depends on the angle of impact of the projectile, the velocity of the projectile, and other parameters. As the impact velocity increases, the optimal thickness of the ceramic increases finds; However, with increasing the angle of impact, the role of the ceramic becomes less, and to achieve the optimal thickness, the thickness of the metal backing layer must be increased.

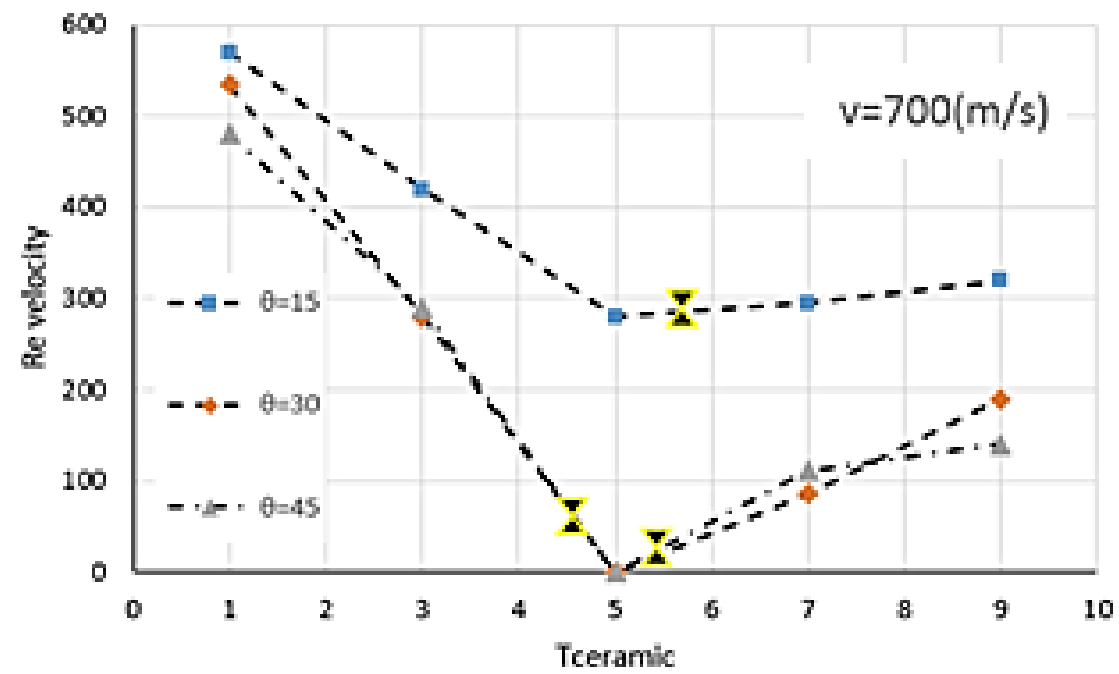

Fig. 11 - Residual velocity in different thicknesses of ceramic 


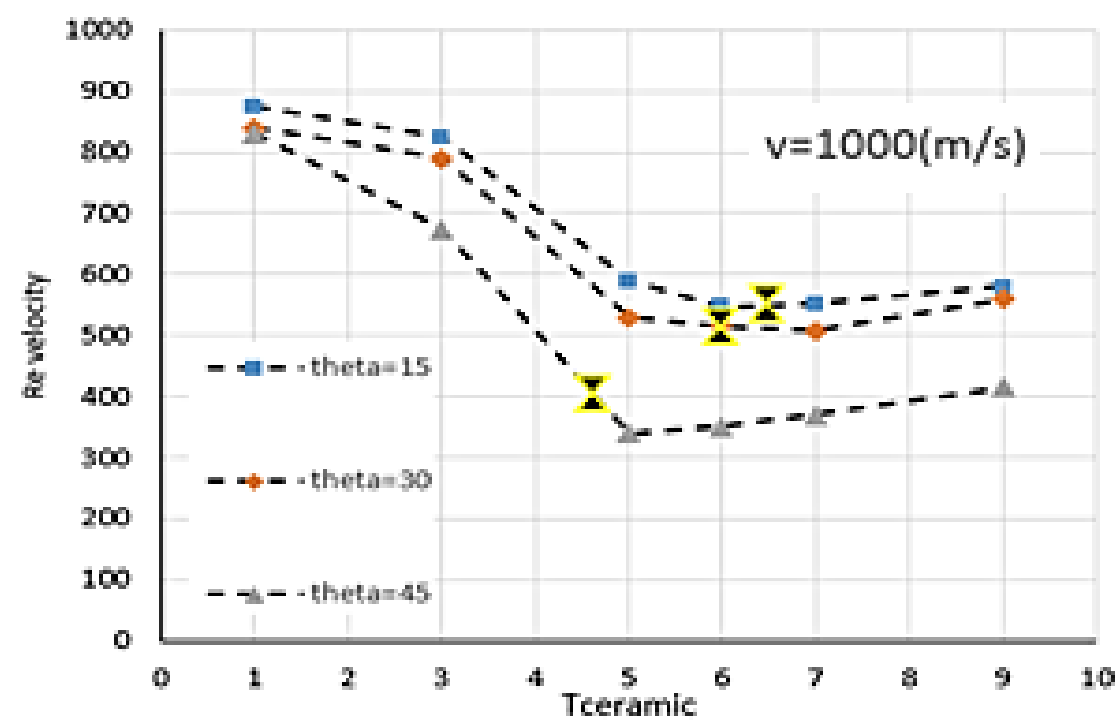

Fig. 12 - Residual velocity in different thicknesses of ceramic

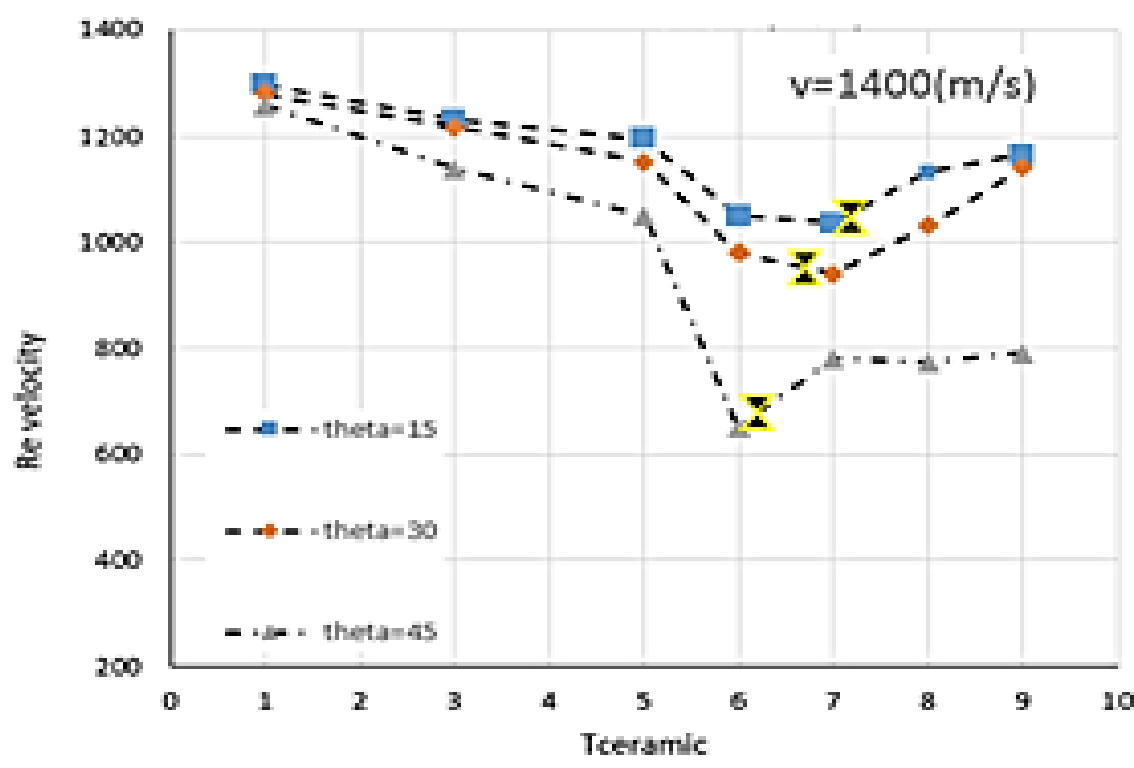

Fig. 13 - Residual velocity in different thicknesses of ceramic

\section{Conclusion}

In this paper, we investigated the explicit numerical solving of the rusting of anti-armored steel projectiles in dealing with alumina ceramic armor with an aluminum support plate. Concerning the simulation and analytical relations, it is clear that with increasing speed, the angle of the buckling angle is increased, and the probability of penetration of the projectile is higher. In the case of an oblique collision at a certain angle, the remaining velocity decreases with increasing target thickness, but this parameter does not affect the directional change angle. As the angle of oblique increases, the amount of penetration in the target decreases. For long rod projectiles, the reduction in kinetic energy at the same collision velocities is not much different for both vertical and oblique collision modes. The erosion of the projectile mass in oblique collisions is less than in vertical collisions at the same time.

\section{Acknowledgement}

The authors would like to thank the Department of Mechanical Engineering, IHU University, Tehran, Iran and Department of Mechanical Engineering, Tehran University, Tehran, Iran. 


\section{References}

[1] G. Jonas and J. A. Zukas, "Mechanics of penetration: analysis and experiment," International Journal of Engineering Science, vol. 16, pp. 879-903, 1978

[2] A. Tate, "A simple estimate of the minimum target obliquity required for the ricochet of a high-speed long rod projectile," Journal of physics. D. Applied Physics, vol. 12, pp. 1825-1829, 1979

[3] N. Gupta, M. Iqbal, and G. Sekhon, "Experimental and numerical studies on the behavior of thin aluminum plates subjected to impact by blunt-and hemispherical-nosed projectiles," International Journal of Impact Engineering, vol. 32, pp. 1921-1944, 2006

[4] Z. Q. Wang, X. J. Tang, X. T. Wang, X. N. Peng, and H. Q. Lv, "Numerical simulation on the oblique penetration of ceramic particles reinforced metal matrix functional armor," in Key Engineering Materials, 2014, pp. 585-588

[5] S. Marina, T. Wolf, R. Nursing. "Numerical Investigations on Ricochet of a Spinstabilised Projectile on Differently Shaped Target Surfaces." 30th International Symposium on Ballistics. 2017

[6] W. Lee, H.-J. Lee, and H. Shin, "Ricochet of a tungsten heavy alloy long-rod projectile from deformable steel plates," Journal of Physics D: Applied Physics, vol. 35 ,p. 2676, 2002

[7] Moslemi Petrudi, A., Kh Vahedi, M. H. Kamyab, and Moslemi Petrudi. "Numerical and Experimental Study of Oblique Penetration of a Blunt Projectile into Ceramic-Aluminum Target." Modares Mechanical Engineering 19, no. 5 (2019): 1253-1263

[8] Liu, Zhaobing, Qian Lei, and Shuaiqi Xing. "Mechanical characteristics of wood, ceramic, metal, and carbon fiber-based PLA composites fabricated by FDM." Journal of Materials Research and Technology 8, no. 5 (2019): 3741-3751

[9] Islam, Md Rushdie Ibne, J. Q. Zheng, and Romesh C. Batra. "Ballistic performance of ceramic and ceramic-metal composite plates with JH1, JH2, and JHB material models." International Journal of Impact Engineering 137 (2020): 103469

[10] Rodríguez Vara, Raúl. "Numerical analysis of the ballistic behavior on aramid aircraft structure." Bachelor's thesis, 2020

[11] Z. Rosenberg, Y. Yeshurun, and M. Mayseless, "On the ricochet of long rod projectiles," in Proc. 11th Int. Symp. Ballistics, 1989

[12] Calder, Clarence Andrew, and W. Goldsmith. "Plastic deformation and perforation of thin plates resulting from projectile impact." International Journal of Solids and Structures 7, no. 7 (1971): 863-881

[13] V. Alekseevskii, "Penetration of a rod into a target at high velocity," Combustion, Explosion, and Shock Waves, vol. 2, pp. 63-66, 1966

[14] A. Tate, "A theory for the deceleration of long rods after impact," Journal of the Mechanics and Physics of Solids, vol. 15 , pp. 387-399, 1967

[15] G. R. Johnson and W. H. Cook, "Fracture characteristics of three metals subjected to various strains, strain rates, temperatures and pressures," Engineering fracture mechanics, vol. 21, pp. 31-48, 1985

[16] Alizadeh, Vahedi Khodadad, "Analytical and Numerical Investigation of Penetration of Conical Projectiles into FML Targets", Quarterly Journal of Aerospace Mechanics, vol. 10th of April 2014 (in Persian) 